\title{
Clinical impact of craniopharyngioma classification based on location origin: a multicenter retrospective study
}

\author{
Wenfu Hu ${ }^{1}$, Binghui Qiu ${ }^{1}$, Fen Mei ${ }^{1}$, Jian Mao ${ }^{1}$, Lizhi Zhou ${ }^{2}$, Fan Liu ${ }^{1}$, Jun Fan ${ }^{1}$, Yi Liu ${ }^{1}$, Ge Wen ${ }^{3}$, \\ Songtao Qi ${ }^{1}$, Yun Bao ${ }^{1}$, Jun Pan ${ }^{1}$ \\ ${ }^{1}$ Department of Neurosurgery, Nanfang Hospital, Southern Medical University, Guangzhou, China; ${ }^{2}$ Department of Biostatistics, School of \\ Biostatistics, Southern Medical University, Guangzhou, China; ${ }^{3}$ Department of Medical Imaging Center, Southern Medical University, Guangzhou, \\ China \\ Contributions: (I) Conception and design: Y Bao, S Qi, J Pan; (II) Administrative support: Y Bao, J Pan; (III) Provision of study materials or patients: \\ J Mao, F Liu, J Fan; (IV) Collection and assembly of data: W Hu, B Qiu, G Wen; (V) Data analysis and interpretation: W Hu, L Zhou; (VI) \\ Manuscript writing: All authors; (VII) Final approval of manuscript: All authors. \\ Correspondence to: Jun Pan, MD, PhD; Yun Bao, MD, PhD. Department of Neurosurgery, Nanfang Hospital, Southern Medical University, \\ Guangzhou, China. Email: sjwkpj@126.com; baoyun519@126.com.
}

Background: An assessment of the clinical impact for craniopharyngiomas (CPs) classification based on origin location has not been reported. The aim of this study was to determine the clinical impact of the site of tumor origin in primary CPs.

Methods: Patients from six national institutions who had undergone resection for primary CP were enrolled. Based on the point of origin and surrounding membranous structures, the location of the tumor origin was labelled as Q, S, or T, where Type Q CPs originated below the diaphragmatic area; Type S CPs originated from Rathke's pouch precursor cells; and Type T CPs originated from the Rathke's pouch precursor cells located above the pars tuberalis. Clinical characteristics, surgical approach, and outcome were evaluated according to the location of the tumor origin.

Results: Among the 529 patients with primary CP, symptoms, age, histopathology type, tumor size, the incidence of hydrocephalus, survival rates, and recurrence-free survival rates were significantly different among tumors originating in different locations. Patients with type T CPs had higher symptom rates of intracranial hypertension and hypothalamic dysfunction, while those with type Q CPs had higher rates of hormone deficits during pre-and post-operative management. Type S CPs were correlated with better outcomes and lower recurrence rates. The location of origin and primary therapy with survival and recurrence in $\mathrm{CP}$ were independent factors for survival and recurrence in multivariate analysis.

Conclusions: The identification of the different location of origin of CPs is of great significance in understanding the relationship between tumors and peripheral tissues. The origin of tumors effects the choice of surgical approach and prognosis.

Keywords: Craniopharyngioma (CP); classification; origin location; outcome

Submitted May 19, 2021. Accepted for publication Jun 28, 2021.

doi: 10.21037/atm-21-2924

View this article at: https://dx.doi.org/10.21037/atm-21-2924

\section{Introduction}

Although craniopharyngioma $(\mathrm{CP})$ is a benign tumor, it is challenging to treat due to the nature of its location relation to peripheral structures (1). Surgical resection is the treatment of choice for $\mathrm{CP}$, and the extent of resection plays a major role in the outcome of surgery. Performing complete resection at presentation offers an optimal choice for tumor control and potential cure with an acceptable morbidity rate (2-4). Hence, a suitable surgical approach can improve survival. The choice of operative approach 
for total resection should be determined according to the mode of tumor growth and as the site of origin of a tumor often has dense adhesions, locating this can predict likely difficulties to be encountered during surgery and aid in the selection of an appropriate surgical approach. Whilst this might lead to differences in the prognosis and recurrence, there have been few investigations to verify this $(4,5)$, and the clinical significance of three different origin types of CP tumors has not yet been clarified. Hence, in this study, we aimed to determine the impact of tumor location origin on the surgical approach, survival, and tumor recurrence in patients undergoing resection of primary CP. We present the following article in accordance with the STROBE reporting checklist (available at https://dx.doi.org/10.21037/ atm-21-2924).

\section{Methods}

\section{Study cohort and clinicopathological variables}

We included all patients who were histologically identified and underwent resection of CPs between March 1997 and December 2012 at six major neurosurgery centers. Patients who underwent surgery for recurrent tumors were excluded due to alterations in the anatomy of the tumor origin site during the first surgery making it difficult to precisely identify. Patients with incomplete records or who were lost to follow-up were also excluded to reduce the bias. All procedures performed in this study involving human participants were in accordance with the Declaration of Helsinki (as revised in 2013). The study was approved by ethics board of Nanfang Hospital, Southern Medical University (No.: NFEC201096). Individual consent for this retrospective analysis was waived.

\section{Follow-up material and basic surgical approach}

All patients underwent exhaustive preoperative and postoperative endocrine examinations involving the pituitary and hypothalamus (6-9). Visual evaluation included corrected and uncorrected visual acuities with visual field examinations in the Ophthalmologic department before and after surgery. All surgical records, image data, and videos were reviewed to help assess the extent of resection and tumor location.

The approaches attempted for gross-total resection in this study included the frontal base interhemispheric, pterional, lateral ventricle, transcallosal, and transsphenoidal. The surgical approach was selected according to the origin of the tumor.

The recurrence-free survival rate, total survival rate, imaging features, and pituitary hypothalamic functions were analyzed using long-term follow-up data. The outcome was divided into good and poor according to the system outlined in the study of Duff et al. as follows (10); good (alive, with functional vision, no major motor deficit in connection with the treatment or tumor progression, Karnofsky Performance Scale score of not less than 80, Katz grade of A, employability for adults at working age, school status of no more than 1 year behind the expected grade for young adults and children, no debilitating emotional or psychological problems), or poor (all patients not meeting the "good" criteria).

\section{Histopathological evaluation and definition of tumor location}

The diagnosis of CP in all patients was confirmed by histology. Preoperative imaging was reviewed by Dr. Jun Pan and Dr. Yun Bao in each case to ascertain the location of the tumor origin for determining the potential for study inclusion in a blinded fashion. If consensus could not be reached, Dr. Songtao Qi was assigned to make the final decision. The pituitary stalk (PS) was divided into four segments in accordance with the folds of the arachnoid sleeve around the pituitary stalk (ASPS) (11). Accordingly, we categorized the tumors into three types $(3,5)$. The origin site of type Q CP is located below the diaphragmatic area and the extra-arachnoidal, intra-arachnoidal, and subarachnoidal segments of the PS as situated above the tumor. The dura mater, arachnoid, pituitary stalk and the third ventricle floor $(3 \mathrm{VF})$ were located above the tumor. These tumors are surrounded by the suprasellar arachnoid and dura mater. Type Q tumors usually demonstrate marked enlargement of the pituitary fossa and a large rounded and symmetrical extension to the suprasellar space. Type S CPs originate from Rathke's pouch precursor cells, that are to the extra- and intra-arachnoidal segments of the PS, and the main body of these tumors is in the suprasellar arachnoid around the PS. The arachnoid and the $3 \mathrm{VF}$ are located above the tumor, the PS is located beside the tumor, the dura mater is below the tumor. Although type $S$ tumors can grow into one or various subarachnoid cisterns, and/or into the pituitary fossa, they tend not to expand the pituitary fossa, and the diaphragm, basal arachnoid, and pituitary can be found below the tumor. If the tumor grows upward, the third ventricle floor can be pushed up. Type T CPs 

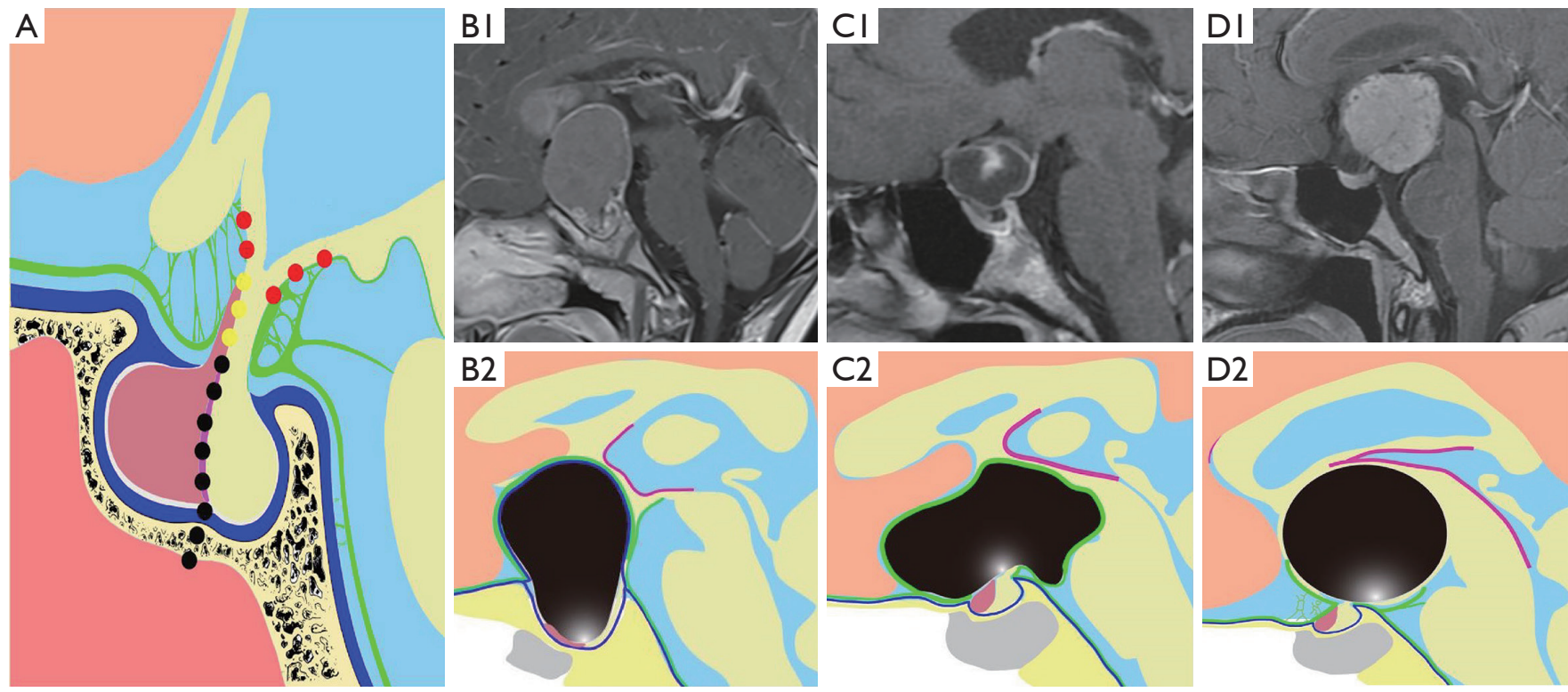

Figure 1 Classification of CPs based on origin location. (A) Several possible sites of tumor origin are indicated as Q (black dots), S (yellow dots), and T (red dots); (B1) preoperative contrast-enhanced MR images of type Q CP; (B2) the relationship between type Q tumors and surrounding structures; (C1) preoperative contrast-enhanced MR images of type S CP; (C2) the relationship between type S tumors and surrounding structures; (D1) preoperative contrast-enhanced MR images of type T CP; (D2) the relationship between type T tumors and surrounding structures. White dots represent the origin point, green lines represent the arachnoid membrane, blue lines represent the dura mater, and violet lines represent the ependyma.

originate from the Rathke's pouch precursor cells, which are at the top of pars the tuberalis. $3 \mathrm{VF}$ is pushed up, and the third ventricle cavity is often occupied by the tumor. The PS, arachnoidal sleeve, and basal arachnoid are beneath type T tumors (Figure 1).

The main differences in the tumors originating from the different sites are as follows: type Q tumors are found below the diaphragm, while type $S$ are located on the extraarachnoidal and intra-arachnoidal segments of the stalk, and type $T$ tumors are situated at the pars tuberalis.

\section{Statistical analysis}

Statistical analysis was performed using IBM SPSS software (version 26.0, SPSS Inc., Chicago, IL, USA). Medians and ranges of continuous variables were compared using the Mann-Whitney $U$ test and categorical variables were compared using Fisher's exact test or Pearson's chi-squared test. The overall survival (OS) was calculated based on the time from surgery until the last follow-up or death. Survival curves were generated using the Kaplan-Meier method and were compared by the log-rank test, and Cox regression analysis was performed to explore possible determinants of outcomes. The value for significance was set at $\mathrm{P} \leq 0.05$.

\section{Results}

\section{Clinical characteristics}

We excluded 213 patients with recurrent tumors and/or radiotherapy and 19 patients with incomplete data or who were lost to follow-up. A total of 529 patients were enrolled from six national institutions. Of these, 209 patients were treated at Nanfang Hospital of Southern Medical University, 87 at the Fuzhou General Hospital of Nanjing Military Area Command, 73 at the Affiliated Hospital of Southwest Medical University, 59 at the First Affiliated Hospital of Fujian Medical University, 51 at the General Hospital of Ningxia Medical University, and 50 at the First Affiliated Hospital of Suzhou University.

The median age of patients with CP was 23 years (range, 2-72 years), and 329 (62.2\%) were men. There were 220 (41.6\%) children and $309(58.4 \%)$ adults when the tumor was detected. The median follow-up period was 83.0 months (range, 28-236 months) excluding the patients who died, who 
Table 1 Preoperative clinical characteristics of patients, stratified according to growth pattern types

\begin{tabular}{|c|c|c|c|c|c|}
\hline Parameter & Type Q (n=142) & Type S ( $n=115)$ & Type T (n=272) & Total $(n=529)$ & $P$ value \\
\hline Mean & $15.18 \pm 14.96$ & $34.90 \pm 18.97$ & $29.30 \pm 19.89$ & $26.73 \pm 19.86$ & $<0.001$ \\
\hline Range & $2-60$ & $3-67$ & $2-72$ & $2-72$ & \\
\hline Children, $\leq 14$ yrs n (\%) & $93(65.5)$ & $24(20.9)$ & $103(37.9)$ & $220(41.6)$ & $<0.001$ \\
\hline \multicolumn{6}{|l|}{ Sex, n (\%) } \\
\hline Male & $93(62.7)$ & $63(55.7)$ & $173(64.7)$ & $329(62.2)$ & 0.168 \\
\hline Female & $49(37.3)$ & $52(44.3)$ & 99 (35.3) & $200(37.8)$ & \\
\hline Tumor size $(\mathrm{cm})$ & $3.7 \pm 1.3$ & $3.9 \pm 1.0$ & $3.6 \pm 1.0$ & $3.7 \pm 1.1$ & 0.028 \\
\hline Range & $17-236$ & $56-196$ & $3-232$ & $3-236$ & \\
\hline \multicolumn{6}{|l|}{ Hydrocephalus, n (\%) } \\
\hline None & $128(90.1)$ & $98(85.2)$ & $139(51.1)$ & $365(69.0)$ & $<0.001$ \\
\hline Yes & $14(9.9)$ & $17(14.8)$ & $133(48.9)$ & $164(31.0)$ & \\
\hline \multicolumn{6}{|l|}{ Tumor consistency, n (\%) } \\
\hline Solid & $18(12.7)$ & $15(13.1)$ & $36(14.0)$ & $71(13.4)$ & 0.695 \\
\hline Mixed & $93(65.5)$ & 77 (66.9) & $164(60.3)$ & $334(63.1)$ & \\
\hline Cystic & $31(21.8)$ & $23(20.0)$ & $70(25.7)$ & $124(23.4)$ & \\
\hline
\end{tabular}

numbered $86(16.3 \%)$ at the time of analysis (Table 1).

\section{QST classification and histopathological features}

A total of 142 patients (26.8\%) had type Q tumors, 115 (21.7\%) had type S tumors, and $272(51.4 \%)$ had type $\mathrm{T}$ tumors, and there were significant differences in the pathological subtypes among the three different types of tumors $(\mathrm{P}<0.001)$. The proportion of patients with adamantinomatous CP (ACP) was 100\% (142/142), 88.7\% $(102 / 115)$, and $86.4 \%(235 / 272)$ among patients with types Q, S, and T tumors, respectively (Table 1).

\section{Symptoms and signs}

The location of the tumor origin and the main symptoms in patients with CP are summarized in Table 2. The tumor location was inferred according to the protocol described above, and the most common symptoms were headache, reduced vision, reduced visual field, and growth retardation. There were significant differences in the frequency of symptoms including headache, nausea or vomiting, diplopia, reduced vision, reduced visual field, seizures, loss of appetite, polyuria, growth retardation, weight gain, altered personality, memory impairment, concentration impairment, and reduced consciousness among the three tumor types.

\section{Treatment and surgical approach}

Of the 529 patients, 403 (76.2\%) were treated with gross total resection (GTR), 81 (15.3\%) with subtotal resection 
Table 2 Presenting symptoms in craniopharyngioma, stratified according to growth pattern types

\begin{tabular}{|c|c|c|c|c|c|}
\hline Symptoms (No.) & Type Q (n=142) & Type S (n=115) & Type T (n=272) & Total $(n=529)$ & $P$ value \\
\hline Nausea or vomiting & $9(6.3)$ & $14(12.2)$ & $89(32.7)$ & $112(21.2)$ & $<0.001$ \\
\hline Diplopia & $32(22.5)$ & $13(11.3)$ & $10(3.7)$ & $55(10.4)$ & $<0.001$ \\
\hline Reduced vision & $75(52.8)$ & $75(65.2)$ & $98(36.0)$ & 248 (46.9) & $<0.001$ \\
\hline Seizures & $5(3.5)$ & $4(3.5)$ & $8(2.9)$ & $17(3.2)$ & $<0.001$ \\
\hline Loss of appetite & $10(7.0)$ & $8(7.0)$ & $8(2.9)$ & $26(4.9)$ & $<0.001$ \\
\hline Weight loss & $4(2.8)$ & $3(2.6)$ & $8(2.9)$ & $15(2.8)$ & 0.984 \\
\hline Polyuria & $17(12.0)$ & $6(5.2)$ & $41(15.1)$ & $64(12.1)$ & 0.025 \\
\hline Insomnia & $5(3.5)$ & $0(0.0)$ & $10(3.7)$ & $15(2.8)$ & 0.117 \\
\hline Altered personality & $1(0.7)$ & $2(1.7)$ & $10(3.7)$ & $13(2.5)$ & 0.008 \\
\hline Memory impairment & $5(3.5)$ & $4(3.5)$ & $30(11.0)$ & $39(7.4)$ & 0.004 \\
\hline Concentration impairment & $1(0.7)$ & $4(3.5)$ & $26(9.6)$ & $31(5.9)$ & 0.001 \\
\hline Reduced consciousness & $0(0.0)$ & $4(3.5)$ & $20(7.4)$ & $24(4.5)$ & 0.002 \\
\hline
\end{tabular}

*, only in age $\leq 14$ years group, $n=220$ (type $Q=93$, type $S=24$, type $T=103$ ).

(STR) without radiation therapy (RT), and $45(8.5 \%)$ with STR with RT. Details of the surgical procedures are summarized in Table 3. The results showed significant differences in the surgical approach among the three different types of tumors $(\mathrm{P}<0.001)$.

\section{Pituitary function and functional outcome}

The cumulative probability for each deficit in all patients is shown in Table 3. There were significant differences in the number of pituitary axes with insufficiencies ( 0 axes, 1-2 axes, and 3-4 axes) among types Q, S, and T tumors at presentation and after surgery $(\mathrm{P}<0.001)$. Of the remaining 336 patients, new anterior hypopituitarism occurred in 94 $(17.8 \%)$, with at least one intact axis preoperatively. The overall clinical outcome was good and poor in $81.9 \%$ and $18.1 \%$ of patients, respectively, and final functional outcomes are shown in Table 3. Patients with type T CPs had a worse outcome than those with type $\mathrm{S}$ and type Q CPs (Table 3).

\section{Recurrent factors for patients with CP}

Among the 529 patients who underwent surgery, 136 were found to have tumor recurrence during follow up and the overall recurrence rate was $25.7 \%$. The median recurrence time was 23 months (range, 1-98 months).

Multivariate analysis was performed to identify the recurrent factors and survival factors for patients with $\mathrm{CP}$, and we selected seven potential candidate factors for analysis including age ( $>14 / \leq 14 \mathrm{y})$, sex (male/female), origin location of the tumor (Q, S, and T), primary therapy (GTR/STR/ STR + RT), pathology (ACP/PCP), calcification status (big/ small $/ \mathrm{no})$, and size status $(>5 / \leq 5 \mathrm{~cm})$.

The recurrence rate was affected by the size $(\mathrm{P}=0.023)$, primary therapy $(\mathrm{P}<0.001)$, and origin location $(\mathrm{P}<0.001)$ in the univariate analyses, and the primary therapy $[\mathrm{P}<0.001 \mathrm{~B}=1.137 \operatorname{Exp}(\mathrm{B})=3.116$, CI $(2.568-3.781)]$, size $[\mathrm{P}=0.001 \mathrm{~B}=0.763 \operatorname{Exp}(\mathrm{B})=2.144$, CI $(1.381-3.329)]$, and origin location $[\mathrm{P}<0.001 \mathrm{~B}=0.396 \operatorname{Exp}(\mathrm{B})=1.485$, CI (1.194-1.847)] were factors for recurrence in the multivariate analysis. The recurrence rate was not affected by age $(\mathrm{P}=0.082)$, sex $(\mathrm{P}=0.343)$, pathology $(\mathrm{P}=0.694)$, and calcification status $(\mathrm{P}=0.615)$ in the univariate analyses.

The recurrence rate after resection was significantly lower for type $\mathrm{S}$ tumors $(13 / 115,11.3 \%)$ than for type Q $(\mathrm{P}=0.002)$, and type $\mathrm{T}$ tumors $(\mathrm{P}<0.001)$. Kaplan-Meier 
Table 3 Presenting symptoms and surgical outcome in craniopharyngioma, stratified according to growth pattern type

\begin{tabular}{|c|c|c|c|c|c|}
\hline Parameter & Type Q (n=142) & Type S $(n=115)$ & Type T (n=272) & Total $(n=529)$ & $P$ value \\
\hline 0 axis & $4(2.8)$ & $51(44.3)$ & $72(26.5)$ & $127(24.0)$ & \multirow[t]{3}{*}{$<0.001$} \\
\hline $1-2$ axes & $35(24.6)$ & $44(38.3)$ & $130(47.8)$ & 209 (39.5) & \\
\hline 3-4 axes & $103(72.5)$ & $20(17.4)$ & $70(25.7)$ & $193(36.5)$ & \\
\hline \multicolumn{6}{|c|}{ Postoperative pituitary dysfunction axes, n (\%) } \\
\hline 0 axis & $1(0.01)$ & $31(27.0)$ & $51(18.6)$ & $83(15.7)$ & \multirow[t]{3}{*}{$<0.001$} \\
\hline $1-2$ axes & $22(15.5)$ & $57(49.6)$ & $121(44.5)$ & $200(37.8)$ & \\
\hline $3-4$ axes & $119(83.8)$ & $27(23.5)$ & $100(36.8)$ & $246(46.5)$ & \\
\hline Frontal base interhemispheric & $50(35.2)$ & $25(21.7)$ & $161(59.2)$ & $236(44.6)$ & \multirow[t]{5}{*}{$<0.001$} \\
\hline Pterion & $54(38.0)$ & $73(63.5)$ & $69(25.4)$ & $196(37.1)$ & \\
\hline Transsphenoidal & $38(26.8)$ & $7(6.1)$ & $6(2.2)$ & $51(9.6)$ & \\
\hline Lateral ventricle & $0(0.0)$ & $2(1.7)$ & $4(1.5)$ & $6(1.1)$ & \\
\hline Transcallosal & $0(0.0)$ & $8(7.0)$ & $32(11.8)$ & $40(7.6)$ & \\
\hline \multicolumn{6}{|l|}{ Functional outcome, n (\%) } \\
\hline Good & $121(85.2)$ & $103(89.6)$ & 209 (76.8) & $433(81.9)$ & \multirow[t]{2}{*}{0.006} \\
\hline Poor & $21(14.8)$ & $12(10.4)$ & $63(23.2)$ & $96(18.1)$ & \\
\hline
\end{tabular}

curves showed that the classification of tumors effectively stratified patient recurrence (Figure $2 A$ ). Recurrences appeared in 39 of 403 patients $(9.7 \%)$ after total resection, in 77 of 81 patients $(95.1 \%)$ after incomplete resection, and in 20 of 45 patients (44.4\%) in incomplete resection plus radiotherapy. GTR was associated with a lower recurrence rate than STR $(\mathrm{P}<0.001)$ or STR with $\mathrm{RT}(\mathrm{P}<0.001)$, and STR with RT was also associated with a lower recurrence rate than STR without RT $(\mathrm{P}<0.001)$. Kaplan-Meier curves showed that the classification of tumors effectively stratified patient recurrence (Figure 2B).

\section{Survival factors for patients with $C P$}

The total OS rate was affected by the primary therapy $(\mathrm{P}<0.001)$, origin location $(\mathrm{P}=0.013)$, and calcification $(\mathrm{P}=0.036)$ in the univariate analyses. The primary therapy $[\mathrm{P}<0.001 \mathrm{~B}=0.795 \operatorname{Exp}(\mathrm{B})=2.215$, CI $(1.684-2.914)]$ and the origin location of the tumor $[\mathrm{P}=0.011 \mathrm{~B}=0.351 \mathrm{Exp}$ (B) $=1.420 \mathrm{CI},(1.085-1.859)]$ were factors for OS in the multivariate analysis. The total OS was lower in type $\mathrm{T}$ participants than in type $\mathrm{Q}(\mathrm{P}=0.026)$ and type $\mathrm{S}(\mathrm{P}=0.020)$ tumors. Kaplan-Meier curves showed that the classification of tumors effectively stratified patient prognosis (Figure 2C). The total OS was higher in GTR tumors than in STR $(\mathrm{P}<0.001)$ and STR with RT $(\mathrm{P}<0.001)$ tumors, and was not significantly different between STR and STR with RT tumors (Figure 2D).

The main reasons for the 86 deaths in our study cohort were tumor progression, hypothalamic-pituitary dysfunction, and intracerebral vascular complications.

\section{Discussion}

$\mathrm{CP}$ is a benign tumor that arises from Rathke's pouch and accounts for $1-4 \%$ of all brain tumors, and the overall incidence of CP is 0.13 per 100,000 person years $(12,13)$. Surgical resection is the first choice for CP followed Yaşargil's view (14). CPs are clinically relevant tumors of the sellar region (15). The management of patients 

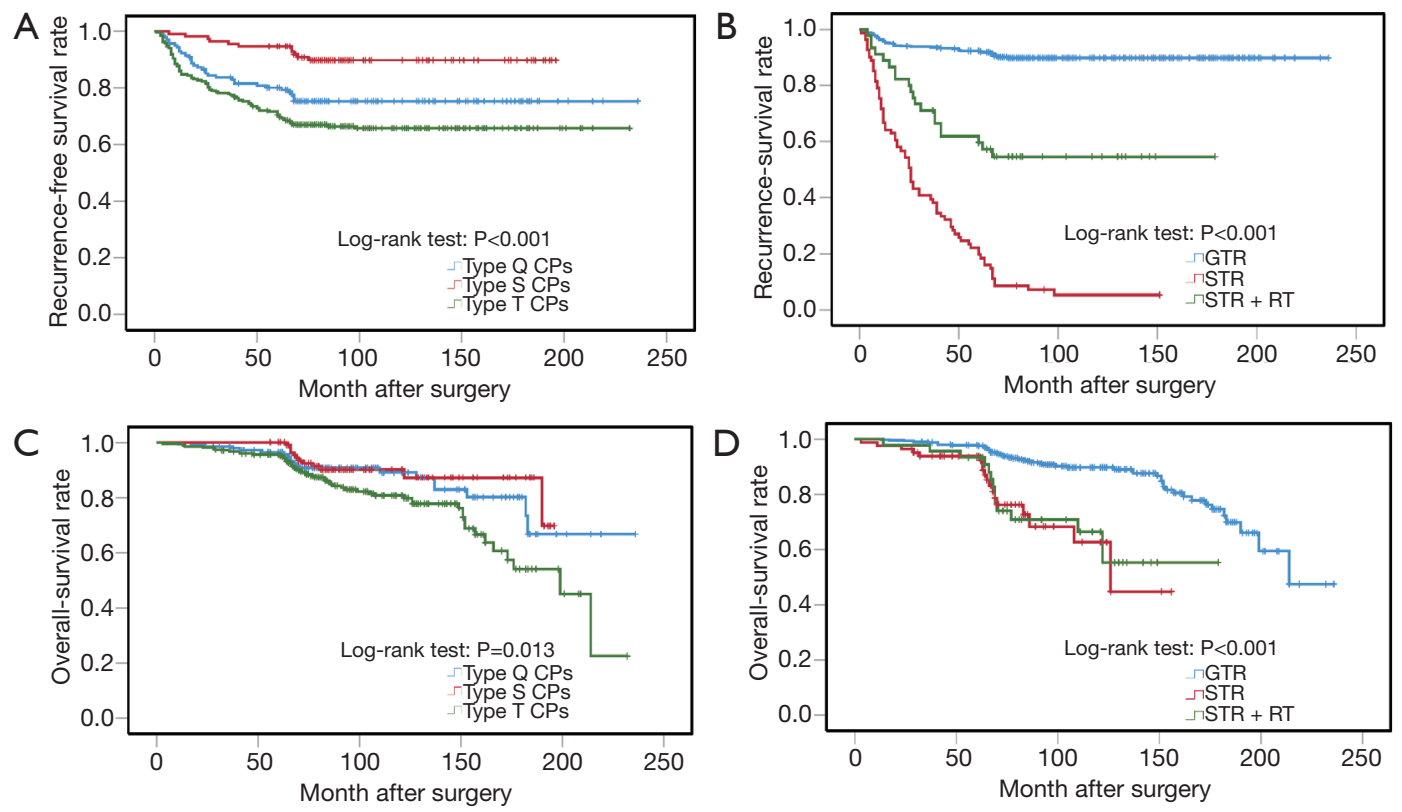

Figure 2 Graph depicting survival times in patients. All $\mathrm{P}$ values are based on log-rank test. (A) The image is related to the recurrence-free survival rate based on tumor origin location; (B) the image is related to the recurrence-free survival rate based on treatment modality; (C) the image is related to the overall-free survival rate based on tumor origin location; (D) the image is related to the overall-free survival rate based on treatment modality in Type T CP. GTR, gross total removal; STR, subtotal removal; RT, radiotherapy.

with $\mathrm{CP}$ is complex and has attracted the attention of many researchers in different fields. To improve the understanding and outcome of CP, several classifications have been put forward including those based on their relationship with the diaphragm or stalk (14), tumor extension (16), endoscopic views of the infundibular region $(17,18)$, and the degree of hypothalamic involvement $(19)$. However, as it is difficult to classify large tumors according to the aforementioned classifications, and all primary CPs were classified based on the location of their origin in the present study.

Although CPs are benign tumors, they are likely to involve the hypothalamic-pituitary axis and surrounding structures, which may limit surgical options to partial resection or lead to severe postoperative complications after total resection, especially severe hypothalamic reactions. To ensure safe surgical resection and avoid severe hypothalamic injury, understanding the pattern of gliosis is essential $(2-4,11)$. The classification based on the origin of the tumor details the relationship of the surrounding structures to CPs and purports to predict and identify the intraoperative anatomical stratification. At the same time, it is not easy to expose the origin of CPs as the lesions have circumferential adhesions with surrounding structures, and the exposure of this area is a priority factor in the choice of surgical approach $(20,21)$.

According to the classification based on the location of the origin point and the relationship between the tumor and arachnoid structures (11), CPs can be divided into three types (5): type Q, type S, and type T. The location of origin of type $\mathrm{Q}$ tumors is below the diaphragm, type $\mathrm{S}$ is on the intra-arachnoidal and extra-arachnoidal segment of the stalk, and type $\mathrm{T}$ is in the pars tuberalis and sub-arachnoidal space. The key differences between type $\mathrm{Q}$ and type $\mathrm{S} / \mathrm{T}$ are the presence of an enlarged pituitary fossa or whether the tumor is above the diaphragm. The main difference between type $\mathrm{S}$ and type T tumors is that the PS sits beside or at the bottom of the tumor, where the tumor extends into the subarachnoid space or the third ventricle, and whether the arachnoid is separated from the tuberoinfundibular and the tumor.

In this study, we analyzed the influence of the location of the tumors origin on clinical characteristics, functional outcome, long-term survival, and recurrence-free survival in patients with primary CP. Our findings show the classification of CP according to the location of tumor origin effectively stratifies the prognosis of patients after undergoing curative resection of the tumor and that 
hypothalamus involvement is correlated with a higher OS rate (22). Type $\mathrm{T}$ tumors were strongly correlated with a higher incidence of hypothalamus involvement, resulting in a worse functional outcome and worse survival rate compared to patients with type $\mathrm{Q}$ and $\mathrm{S}$ tumors.

Significant endocrine dysfunction is seen in most patients with CP, and in this study, we found that pituitary dysfunction in $\mathrm{CP}$ was largely related to the location of its origin point. It is common to find three or more hormone deficits in patients with type $\mathrm{Q}$ tumors, which might be due to the direct effect of the pituitary on tumor compression and inflammation (23). Endocrine replacement after GTR also offers a chance of cure in type Q tumors. As Type $S$ tumors mainly grow in the subarachnoid space, which usually has a mild or moderate effect on the PS and 3VF, pituitary dysfunction in patients is usually not serious. Type $\mathrm{T}$ tumors usually extend to the $3 \mathrm{VF}$ and occupy the space of the third ventricle, resulting in hypothalamus damage. Owing to this, hypothalamic-pituitary dysfunction occurs frequently in this tumor type.

In addition, we found that the symptoms, age, histopathology type, tumor size, and incidence of hydrocephalus were significantly different in the different types of tumors. Patients with type Q tumors were more likely to be younger compared to those with type $S$ and $T$ tumors. Several studies have described the symptoms of CPs $(7,24,25)$, which include headache, nausea, vomiting, and symptoms related to hypothalamic function. These symptoms were more frequently observed in patients with type T tumors, which may be associated with intracranial hypertension. Memory deficits, behavioral abnormalities, and psychosocial impairment frequently occur early after the diagnosis of CP $(8,26)$, and were especially seen in patients with type T CP in the present study, while reduced vision and visual field symptoms were more frequently observed in patients with type $\mathrm{S}$ and $\mathrm{Q}$ tumors than in those with type $\mathrm{T}$ tumors.

As some authors have reported cases in which an erroneous classification of the type of CPs has resulted in an incorrect surgical approach $(15,27,28)$, it is particularly important to consider the classification of the tumor based on its origin site (29), before surgery. The initial site of the tumor is usually the closest to the surrounding tissue, and the relationship between the tumor and the surrounding tissue (such as the hypothalamus) differs in different tumor types. The transsphenoidal approach is the most appropriate corridor to treat some types of $\mathrm{Q}$ tumors, as it avoids injury to the optic chiasm and third ventricle. The continuity of the $3 \mathrm{VF}$-stalk-pituitary gland can be well protected in type $S$ tumors via extra-axial approaches, while for type $T$ tumors, if the upper edge of the tumor exceeds the mass intermedia, the trans-lamina terminalis approach can be selected to allow for additional exposure of the tumor. In addition, the classification of $\mathrm{CP}$ according to its site of origin can predict the adherent point and possible difficulties to during the course of the surgery. It is also important to predict the risk of CP adherence to the hypothalamus (29). Type $\mathrm{T}$ tumors are a strong predictor of adherence to the hypothalamus because of their origin point and radical resection becomes highly challenging because of their tenacious adherence to the infundibulo-tuberal region and the hypothalamus. Radical resection of type $\mathrm{S}$ tumors is easier than the other two tumor types, as well as a Grade V tumor in the classification system based on vertical extension (16), and the arachnoid between the tumor and $3 \mathrm{VF}$ should be a reliable boundary.

Recently, progress in molecular biology has unravelled creative, potentially druggable molecular pathways in CPs that provide new prospective therapeutic options. The treatment of ACP with vismodegib, a sonic hedgehog pathway inhibitor, results in a substantial reduction in median survival in murine models and further research is needed to effectively translate these laboratory findings to clinical settings (30). BRAF inhibitors have been demonstrated to show effectiveness against BRAF-mutated papillary CP. Related drugs are already in clinical trials, but are currently only used for recurrent CPs $(31,32)$.

\section{Limitations}

The limitations of this study include its retrospective nature and associated biases. However, CPs are relatively rare, and the study is one of the largest series to analyze the tumor. Moreover, the current data were obtained from six highvolume centers in the East, South, West, and North of China. The proposed definitions of origin location offer a simple and reliable method to evaluate the prognosis of patients with CP.

\section{Conclusions}

Identification of the tumor origin site is crucial in the management of primary CP. The classification of primary $\mathrm{CP}$ into Q, S, and T tumors provides a useful tool for selecting the surgical approach and predicting its outcome. 


\section{Acknowledgments}

The authors wish to thank Dezhi Kang (The First Affiliated Hospital, Fujian Medical University, Fujian province, China), Ligang Chen (Affiliated Hospital of Southwest Medical University, Sichuan province, China), Zongzheng Li (General Hospital of Ningxia Medical University, Ningxia province, China), Shousen Wang (Fuzhou General Hospital of Nanjing Military Area Command, Fujian Province, China), and Zhengquan Yu (The First Affiliated Hospital to Suzhou University, Jiangsu Province, China) for providing critical clinical data.

Funding: This work was supported by the National Natural Science Foundation of China (81972352); National Natural Science Foundation of Guangdong Province of China (2017A020215048); Clinical Research Startup Program of Southern Medical University by High-level University Construction Funding of Guangdong Provincial Department of Education (LC2016PY012); and Clinical Research Program of Nanfang Hospital, Southern Medical University (2018CR029).

\section{Footnote}

Reporting Checklist: The authors have completed the STROBE reporting checklist. Available at https://dx.doi. org/10.21037/atm-21-2924

Data Sharing Statement: Available at https://dx.doi. org/10.21037/atm-21-2924

Conflicts of Interest: All authors have completed the ICMJE uniform disclosure form (available at https://dx.doi. org/10.21037/atm-21-2924). The authors have no conflicts of interest to declare.

Ethical Statement: The authors are accountable for all aspects of the work in ensuring that questions related to the accuracy or integrity of any part of the work are appropriately investigated and resolved. All procedures performed in this study involving human participants were in accordance with the Declaration of Helsinki (as revised in 2013). The study was approved by ethics board of Nanfang Hospital, Southern Medical University (No.: NFEC201096). Individual consent for this retrospective analysis was waived.

Open Access Statement: This is an Open Access article distributed in accordance with the Creative Commons Attribution-NonCommercial-NoDerivs 4.0 International License (CC BY-NC-ND 4.0), which permits the noncommercial replication and distribution of the article with the strict proviso that no changes or edits are made and the original work is properly cited (including links to both the formal publication through the relevant DOI and the license). See: https://creativecommons.org/licenses/by-nc-nd/4.0/.

\section{References}

1. Zoicas F, Schöfl C. Craniopharyngioma in adults. Front Endocrinol (Lausanne) 2012;3:46.

2. Elliott RE, Wisoff JH. Successful surgical treatment of craniopharyngioma in very young children. J Neurosurg Pediatr 2009;3:397-406.

3. Liu Y, Qi ST, Wang CH, et al. Pathological Relationship Between Adamantinomatous Craniopharyngioma and Adjacent Structures Based on QST Classification. J Neuropathol Exp Neurol 2018;77:1017-23.

4. Qi S, Pan J, Lu Y, et al. The impact of the site of origin and rate of tumour growth on clinical outcome in children with craniopharyngiomas. Clin Endocrinol (Oxf) 2012;76:103-10.

5. Bao Y, Pan J, Qi ST, et al. Origin of craniopharyngiomas: implications for growth pattern, clinical characteristics, and outcomes of tumor recurrence. J Neurosurg 2016;125:24-32.

6. Castro-Dufourny I, Carrasco R, Pascual JM. Hypothalamic obesity after craniopharyngioma surgery: Treatment with a long acting glucagon like peptide 1 derivated. Endocrinol Diabetes Nutr 2017;64:182-4.

7. Ghirardello S, Hopper N, Albanese A, et al. Diabetes insipidus in craniopharyngioma: postoperative management of water and electrolyte disorders. J Pediatr Endocrinol Metab 2006;19 Suppl 1:413-21.

8. Molitch ME, Clemmons DR, Malozowski S, et al. Evaluation and treatment of adult growth hormone deficiency: an Endocrine Society clinical practice guideline. J Clin Endocrinol Metab 2011;96:1587-609.

9. Verbalis JG, Goldsmith SR, Greenberg A, et al. Hyponatremia treatment guidelines 2007: expert panel recommendations. Am J Med 2007;120:S1-21.

10. Duff J, Meyer FB, Ilstrup DM, et al. Long-term outcomes for surgically resected craniopharyngiomas. Neurosurgery 2000;46:291-302; discussion 302-5

11. Qi S, Lu Y, Pan J, et al. Anatomic relations of the arachnoidea around the pituitary stalk: relevance for 
surgical removal of craniopharyngiomas. Acta Neurochir (Wien) 2011;153:785-96.

12. Giordano M, Zaed I. Value of magnetic resonance imaging in predicting BRAF mutation in craniopharyngiomas. Transl Cancer Res 2019;8:S97-8.

13. Louis DN, Perry A, Reifenberger G, et al. The 2016 World Health Organization Classification of Tumors of the Central Nervous System: a summary. Acta Neuropathol 2016;131:803-20.

14. Yaşargil MG, Curcic M, Kis M, et al. Total removal of craniopharyngiomas. Approaches and long-term results in 144 patients. J Neurosurg 1990;73:3-11.

15. Lubuulwa J, Lei T. Pathological and Topographical Classification of Craniopharyngiomas: A Literature Review. J Neurol Surg Rep 2016;77:e121-7.

16. Samii M, Bini W. Surgical treatment of craniopharyngiomas. Zentralbl Neurochir 1991;52:17-23.

17. Jeswani S, Nuño $M, W u$ A, et al. Comparative analysis of outcomes following craniotomy and expanded endoscopic endonasal transsphenoidal resection of craniopharyngioma and related tumors: a single-institution study. J Neurosurg 2016;124:627-38.

18. Kassam AB, Gardner PA, Snyderman CH, et al. Expanded endonasal approach, a fully endoscopic transnasal approach for the resection of midline suprasellar craniopharyngiomas: a new classification based on the infundibulum. J Neurosurg 2008;108:715-28.

19. Puget S, Garnett M, Wray A, et al. Pediatric craniopharyngiomas: classification and treatment according to the degree of hypothalamic involvement. J Neurosurg 2007;106:3-12.

20. Pan J, Qi S, Lu Y, et al. Intraventricular craniopharyngioma: morphological analysis and outcome evaluation of 17 cases. Acta Neurochir (Wien) 2011;153:773-84.

21. Steno J, Malácek M, Bízik I. Tumor-third ventricular relationships in supradiaphragmatic craniopharyngiomas: correlation of morphological, magnetic resonance imaging, and operative findings. Neurosurgery 2004;54:1051-58; discussion 1058-60.

Cite this article as: $\mathrm{Hu}$ W, Qiu B, Mei F, Mao J, Zhou L, Liu F, Fan J, Liu Y, Wen G, Qi S, Bao Y, Pan J. Clinical impact of craniopharyngioma classification based on location origin: a multicenter retrospective study. Ann Transl Med 2021;9(14):1164. doi: 10.21037/atm-21-2924
22. Sterkenburg AS, Hoffmann A, Gebhardt U, et al. Survival, hypothalamic obesity, and neuropsychological/ psychosocial status after childhood-onset craniopharyngioma: newly reported long-term outcomes. Neuro Oncol 2015;17:1029-38.

23. Daubenbüchel AM, Müller HL. Neuroendocrine Disorders in Pediatric Craniopharyngioma Patients. J Clin Med 2015;4:389-413.

24. Larijani B, Bastanhagh MH, Pajouhi M, et al. Presentation and outcome of 93 cases of craniopharyngioma. Eur J Cancer Care (Engl) 2004;13:11-5.

25. Nielsen EH, Jørgensen JO, Bjerre P, et al. Acute presentation of craniopharyngioma in children and adults in a Danish national cohort. Pituitary 2013;16:528-35.

26. Muller HL. Childhood craniopharyngioma. Recent advances in diagnosis, treatment and follow-up. Horm Res 2008;69:193-202.

27. Fukushima T, Hirakawa K, Kimura M, et al. Intraventricular craniopharyngioma: its characteristics in magnetic resonance imaging and successful total removal. Surg Neurol 1990;33:22-7.

28. Pascual JM, González-Llanos F, Barrios L, et al. Intraventricular craniopharyngiomas: topographical classification and surgical approach selection based on an extensive overview. Acta Neurochir (Wien) 2004;146:785-802 .

29. Prieto R, Pascual JM, Subhi-Issa I, et al. Predictive factors for craniopharyngioma recurrence: a systematic review and illustrative case report of a rapid recurrence. World Neurosurg 2013;79:733-49.

30. Carreno G, Boult JKR, Apps J, et al. SHH pathway inhibition is protumourigenic in adamantinomatous craniopharyngioma. Endocr Relat Cancer 2019;26:355-66.

31. Aylwin SJ, Bodi I, Beaney R. Pronounced response of papillary craniopharyngioma to treatment with vemurafenib, a BRAF inhibitor. Pituitary 2016;19:544-6.

32. Brastianos PK, Taylor-Weiner A, Manley PE, et al. Exome sequencing identifies BRAF mutations in papillary craniopharyngiomas. Nat Genet 2014;46:161-5.

(English Language Editor: B. Draper) 